\title{
Cash Transfers in adolescence: a developmental perspective
}

Julia Ruiz Pozuelo ${ }^{12}$, Alan Stein ${ }^{3}$, Sarah-Jayne Blakemore ${ }^{4}$, Kathleen Kahn ${ }^{3}$, Stefan Dercon ${ }^{2}$, Audrey Pettifor ${ }^{5}$

${ }^{1}$ Department of Psychiatry, University of Oxford

${ }^{2}$ Centre for the Study of African Economies, Blavatnik School of Government, University of Oxford

${ }^{3}$ MRC/Wits Rural Public Health and Health Transitions Research Unit (Agincourt), School of Public Health, Faculty of Health Sciences, University of Witwatersrand

${ }^{4}$ Department of Psychiatry, University of Cambridge

${ }^{5}$ Department of Epidemiology, University of North Carolina at Chapel Hill

Low-income and middle-income countries have increasingly relied on cash transfer programmes to expand social protection among the most poor and vulnerable people. Many of these cash transfer programmes have given cash directly to adolescents to incentivise behaviour that improve a range of health and social outcomes. The evidence for the effectiveness of these programmes has been mixed. Some studies show cash transfer to be effective in promoting desired behaviours among adolescentssuch as school participation and reducing sexually transmitted infections - whereas others find no evidence for an effect. One possible reason for these mixed results is that cash transfer programmes targeting adolescents have not taken into account the many biological, cognitive, and social changes that occur during this transitional period, thus failing to incorporate a developmental perspective in the design.

Adolescence (between 10-24 years) is the period when individuals start to develop their self-identity, acquire the necessary skills to navigate future challenges, and make important decisions about their health, education, relationships, and entrance into the labour market-within a context of diminishing parental control and heightened peer influence. In this Comment we discuss two critical processes that are characteristic of adolescence and are likely to play an important role in determining whether a cash transfer programme is effective. We conclude with some specific steps that these programmes could take to address these developmental factors.

First, adolescence is a period of social reorientation. Compared with children, adolescents start spending more time with peers than with family members, placing more value on what their peers think about them and establishing complex peer relationships. During this period, adolescents become increasingly selfconscious, sensitive to social exclusion, and influenced by their peers. Second, adolescents are more likely to be involved in risky behaviours and sensation seeking compared with children or adults, especially when with peers. ${ }^{1}$ At the same time, the brain undergoes substantial and protracted development throughout adolescence and into early adulthood, and therefore adolescence is considered a period of heightened neuroplasticity, when brain development is particularly influenced by environmental experience. ${ }^{2}$ Adolescence is thus a period of both vulnerability and opportunity for intervention.

These critical processes make it likely that cash transfer programmes have different effects depending on the adolescent's developmental trajectory and social context. A conditional cash transfer programme that aimed to reduce HIV risk among young women (13-20 years) in rural South Africa found differences in how the money was spent depending on the participant's age. Older adolescents ( $\geq 17$ years) spent relatively more cash on items to enhance their personal appearance such as makeup, cosmetics, hairdressing, and school uniform than did those in early adolescence (13-16 years). Most participants stated that the cash allowed them to fit in and gain acceptance and respect within their peer group. ${ }^{3}$ Similarly, a cash transfer 
programme undertaken in Tanzania with out-of-school young women (15-23 years at baseline) found that, as participants grew older, fewer reported spending the money on self-care items and more used it to start new businesses, invest in livestock, or save for future medical expenses. Older adolescents also reported being more concerned about having to care for their family, and less so about needing to fit in with peers. ${ }^{4}$

One way to reduce risk-taking among adolescents is to make the trade-offs involved in their decisions more salient. Evidence shows that interventions providing this information (eg, on returns to education, safe sex practices, or financial education) can be cost effective. A study in the Dominican Republic showed that, when adolescents were provided information about the benefits of education, they completed $0 \cdot 20-0 \cdot 35$ more years of school over the next 4 years than those who did not receive such information. ${ }^{5}$ An intervention in Kenya that informed adolescents of the risk of older partners reduced pregnancy by $28 \%$ in 1 year. ${ }^{6}$ The abovementioned cash transfer programme in Tanzania included financial education, which enabled adolescents to set realistic and productive financial goals and to discuss different ways to stay on track to achieve these goals. ${ }^{7}$ Some interventions have integrated so-called behavioural nudges (such as sending reminders to participants via text messages) to support adolescents in meeting their goals. These findings show that giving information and correcting misconceptions may be just as important as providing cash.

Consistent with the evidence that adolescents are strongly influenced by peers, several studies have shown that interventions delivered by peer mentors are more likely to affect behaviour. ${ }^{7}$ For example, a study in Botswana evaluating the delivery of information about safe sex practices to adolescent girls found that the intervention reduced pregnancy when delivered by peer mentors but had no effect when delivered by older teachers. ${ }^{8}$

Peer mentors could also help adolescents to deal with self-esteem and peer influence. Evidence from South Africa showed that young women (13-20 years) with low self-esteem were more likely to engage in transactional sex, and that adolescents who perceived that their friends were having sex were more likely to be positive for herpes simplex virus type 2 or HIV. ${ }^{9}$ This finding is consistent with evidence showing that peer pressure and the fear of social rejection might induce adolescents to engage in risky decisions, despite the associated health risks. ${ }^{1}$

Adolescence is a period of change. Social changes that facilitate new relationships and risks that foster creativity are necessary to successfully transition to adulthood. ${ }^{10}$ But adolescence is also a time of increased vulnerability, which can result in negative life outcomes such as early pregnancy, sexually transmitted diseases, mental illness, or substance misuse. Giving cash to adolescents has many benefits, such as offering independence and autonomy from their parents, developing their own identity, and making an important step towards financial independence. ${ }^{3}$ Adjusting cash transfer programmes to incorporate a developmental perspective in their design has the potential to alleviate material constraints and promote successful lifecourse choices for adolescents.

The authors declared no conflicts of interest.

\section{References}

1. Blakemore S-J, Mills KL. Is Adolescence a Sensitive Period for Sociocultural Processing? Annu Rev Psychol. 2014;65(1):187-207. 
2. Tamnes CK, Herting MM, Goddings A-L, Meuwese R, Blakemore S-J, Dahl RE, et al. Development of the Cerebral Cortex across Adolescence: A Multisample Study of Inter-Related Longitudinal Changes in Cortical Volume, Surface Area, and Thickness. J Neurosci Off J Soc Neurosci. 2017 22;37(12):3402-12.

3. MacPhail C, Khoza N, Selin A, Julien A, Twine R, Wagner RG, et al. Cash transfers for HIV prevention: what do young women spend it on? Mixed methods findings from HPTN 068. BMC Public Health. 2018 Dec 11;18(1):10.

4. Wamoyi J, Balvanz P, Gichane MW, Maman S, Mugunga S, Majani E, et al. Decision-making and cash spending patterns of adolescent girls and young women participating in a cash-transfer intervention in Tanzania: Implications for sexual health. Glob Public Health. 2019 Dec 6;1-11.

5. Jensen R. The (Perceived) Returns to Education and the Demand for Schooling. Q J Econ. 2010 May $1 ; 125(2): 515-48$.

6. Dupas P. Do Teenagers Respond to HIV Risk Information? Evidence from a Field Experiment in Kenya. Am Econ J Appl Econ. 2011 Jan;3(1):1-34.

7. Pettifor A, Wamoyi J, Balvanz P, Gichane MW, Maman S. Cash plus: exploring the mechanisms through which a cash transfer plus financial education programme in Tanzania reduced HIV risk for adolescent girls and young women. J Int AIDS Soc [Internet]. 2019 Jul 22 [cited 2019 Oct 3];22(Suppl Suppl 4). Available from: https://www.ncbi.nlm.nih.gov/pmc/articles/PMC6643075/

8. Angrist N, Matshaba M, Gabaitiri L, Anabwani G. Revealing a safer sex option to reduce HIV risk: a cluster-randomized trial in Botswana. BMC Public Health. 2019 May 21;19(1):610.

9. Giovenco D, Pettifor A, Hughes j., MacPhail C, Wagner R, Kahn K. Self-esteem as an indicator of transactional sex among young women in rural South Africa. AIDS Behav [Internet]. 2019 [cited 2019 Oct 3]; Available from: https://programme.aids2018.org/Abstract/Abstract/6338

10. Duell N, Steinberg L. Positive Risk Taking in Adolescence. Child Dev Perspect. 2019;13(1):48-52. 